\title{
ARCHIV FÜR
}

GARTENBAU

Z

BAND $31 \cdot 1983 \cdot$ HEFT 8

ISSN 0003-908X

Arch. Garțenbau, Berlin 31 (1983) 8, S. 367-440

EVP 5,- M 
Zoltechrift „Archiv for Gartenban“"

Herausgeber: Akademie der Landwirtschaftswissenschaften

der Deutschen Demokratischen Republik

DDR - 1086 Berlin, Krausenstraße 38/39.

Verlag: Akademie-Verlag, DDR - 1086 Berlin, Leipziger Straße 3-4, PF-Nr. 1233;

Fernruf: 2236221 oder 2236229 , Telex-Nr : 114420 ;

Bank: Staatabank der DDR, Berlin, Kto.-Nr.: 6836-26-20712.

Chefredakteur: Prof. Dr. sc. Wolfgang Frhrmans, Institut für Obstforschung Dreaden-Pillnitz der AdL, DDR - 8057 Dresden, Pillnitzer Platz 2.

Redaktionskollegium: Prof. Dr. sc. H. Bochow, Berlin; Dr. B. ExGkL, GroBbeeren; Prof. Dr. sc. H. Fröhuch, Großbeeren; Prof. Dr. F. Göhler, Großbeeren; Prof. Dr. sc. H.-G. Kauryasn, Berlin; Prof. Dr. sc. H. Krgler, Abchersleben;

Prof. Dr. sc. Dr. h. c. S. Kr

Prof. Dr. habil. G. Stoule, Halle; Prof. Dr sc. G. Voak, Großbeeren; Dr. sc. R. Wejchoud, Quedlinburg;

Dr. H. ZIMMERMans; Nossen.

Anschrift der Redaktion: Institut für Obstforschung Dresden-Pillnitz der AdL, nArchiv für Gartenban ${ }^{\prime}$, DDR - 8057 Dresden, Pillnitzer Platz 2.

Veroffentlicht unter der Lizenznummer 1276 des Presseamtes beim Vorsitzenden des Ministerrates der Deutschen Demokratischen Republik.

Gesamtherstellung: VEB Druckerei „Gottfried Wilheln Leibniz ${ }^{4}$, DDR - 4450 Gräfenhainichen.

Erschelnứgsweise: Die Zeitschrift „Archiv für Gartenbau“ erscheint jährlich in einem Ban̄ mit 8 Heften. Das letzte Heft eines Bandes enthălt Inhalts-, Autoren- und Sachverzeichnils. Bezugspreis eines Bandes 160,- M zuzäglich.Versandspesen (Preis für die DDk 40,- M). Preis je Heft 20,- M (Preis für die DDR 5,- M).

Bestellnummer dieses Heftes: 1039/31/8.

Urheberrecht: Die Rechte über die in dieser Zeitschrift abredruckten Arbeiten gehen ausschlieBlich an die Akademie đer Landwirtschaftswissenschaften der Deutschen Demokratischen RepubHk über. Ein Nachdruck in anderen Zeitschriften oder eine UUbersetzang in andere Sprachen bedarf der Genehmigung der Akademie, ausgenommen davon bleibt der Abdruck von Zusammenfassungen. Kein anderer Tetl dieser Zeitschrift darf in irgendeiner Form - durch Photokopie, Mikrofilm oder ein anderes Verfahren - ohne schriftliche Genehmigung der Akademie reproduziert werden.

All rights reserved (Including those of translation into foreign languages). No part of this issue, except the summaries may' be reproduced in any form, by photoprint, microfilm or any other means, without written permission from the pub. Hshers.

(C) 1983 by Akademie-Verlag. Printed in the German Dernocratic Republic.

AN (EDV) 51515

Bestellungen sind $\mathbf{z u}$ richten

- in der $\widetilde{D} D R$ an den Postzeitungsvertrieb unter Angabe der Kundennummer des Bestellenden oder an den AKADEMIE-Verlag, DDR - 1086 Berlin, Telpziger Straßè 3-4, PF-Nr. 1233;

- im sozialistischen Ausland an eine Buchhandlung für fremdaprachige Literatur oder an den zustāndigen Postzeitangsvertileb;

- in der BRD und Berlin (West) an eine Buchhandlung oder an die Auslieferungsstelle KUNST UND WISSEN, Erich Bieber OHG, Wilhelmstraße 4-6, D - 7000 Stuttgart 1;

- in den abrigen vesteuropdischen Ländern an eine Buchhandlang oder an die Auslieferungsatelle KUNST UND WISSEN, Erich Bieber GmbH, Dufourstraße 51, CH - 8008 Zürich;

- im Hbrigen Ausland an den Internationalen Buch- und Zeitschriftenhandel; den Buchexport, Volkseigener Außenhandelsbetrieb der Deutschen Demokratischen Republik, DDR - 7010 Lolpzig, Postfach 160; oder an der' ARADEMIE-VERLAG, DDR - 1086 Berlin, Leipziger Straße 3-4, PF-Nr. 1233. 